\title{
Heparin-Bonded Viabahn and Drug-Eluting Bare Metal Zilver (PTX) Sandwich Stents in Femoral Chronic Total Occlusion: A Retrospective Review of Mid-Term and Long-Term Outcomes
}

\author{
Amir Awwad, ${ }^{1}$ Waleed Al-Obaydi, ${ }^{2}$ Yao Pey Yong, ${ }^{1}$ and Said B. Habib ${ }^{2}$ \\ ${ }^{1}$ Division of Radiological Sciences, School of Medicine, University of Nottingham, Queen's Medical Centre, B Floor, West Block, \\ Derby Road, Nottingham NG7 2UH, UK \\ ${ }^{2}$ Department of Interventional Radiology, Nottingham University Hospitals NHS Trust, Queen's Medical Centre, B Floor, West Block, \\ Derby Road, Nottingham NG7 2UH, UK
}

Correspondence should be addressed to Said B. Habib; said.habib@nuh.nhs.uk

Received 28 September 2014; Accepted 19 December 2014

Academic Editor: Hironori Nakagami

Copyright (c) 2015 Amir Awwad et al. This is an open access article distributed under the Creative Commons Attribution License, which permits unrestricted use, distribution, and reproduction in any medium, provided the original work is properly cited.

\begin{abstract}
Articles describe a retrospective cohort study to assess the feasibility of reducing neointimal hyperplasia following interventions in chronic total occlusive peripheral vascular disease. The "sandwich" technique implemented is a novel modified angioplasty technique that combines heparin-bonded (Viabahn) stents with drug-eluting stents (Zilver PTX) deployed during arterial recanalisation. A review of 46 cases underwent this procedure at our institution as a limb saving procedure is conducted to determine mid-long-term patency outcomes and identify any subsequent interventions. Followup demonstrates favourable midlong-term patency in $50 \%$ of TASC-C cases. No documented postprocedural complications were noted with unrelated observed mortality rates in either TASC-C or TASC-D subgroups. An observed advantage could be gained by using this new stenting technique via the subintimal recanalisation route. Albeit not statistically significant, type II diabetics and patients on aspiring had better sustained patency angioplasties. The sandwich technique has been shown to effectively offer symptoms relief and reduce total duration of hospital stay. Study findings support the need to evaluate the long-term efficacy through a larger longitudinal prospective study. However, to overcome the encountered study limitations, a well-structured methodology for comparison with conventional angioplasty techniques is essential to assess the synergetic potential of the sandwich technique.
\end{abstract}

\section{Introduction}

Peripheral arterial disease affects $15-20 \%$ of aging population above 75 years of age [1]. Due to the low morbidity and mortality compared with open surgery, endovascular therapy is a desirable primary option [2]. Percutaneous transarterial angioplasty (PTA) for revascularization of diseased superficial femoral artery (SFA) can achieve initial technical success rates above $95 \%$ with a low risk for complications [3]. Long segment and more extensive peripheral arterial disease demonstrate poorer outcome following PTA with a restenosis rate above $70 \%$ in the first year [4]. There are improved clinical outcomes and patency with bare nitinol (NiTi) stent (BNS) placement in comparison to balloon PTA alone [5-11]. However, intimal hyperplasia particularly on either end of the stent graft represents a pathological challenge to this treatment which eventually leads to disease recurrence (restenosis) [12].

In recent years, development of a balloon or stent capable of delivering a pharmaceutical agent (drug-eluting) to prevent inflammation and intimal hyperplasia is achieved with the only safe and effective drug available, paclitaxel. The method of delivery in these devices depends on stent/balloon design and drug kinetics (coating versus adsorption). While duration of contact with the vessel wall (balloon delivery) may be brief (1-3 minutes), the effect of retained drug continues over several weeks [13]. Efficacy and better outcome measures following the use of drug covered balloons (DCBs) were proven when compared to plain balloon PTA $[14,15]$. Currently, DESs have been successfully shown to treat longer 
and difficult CTO lesions in femoropopliteal disease as demonstrated in few concluded and current trials [16, 17].

Zilver PTX (Cook Medical, Bloomington, IN) is a NiTi drug-eluting stent that has been tested in a large international randomized trial comparing Zilver PTX with bare Zilver stent by recruiting two randomization protocols. Their recent outcome of 3 years of data has shown a maintained reduction of restenosis rate by $50 \%$, which has granted this stent FDA and $\mathrm{CE}$ approval to be the only NiTi based self-expanding drugeluting stent $[8,18-20]$.

Another FDA and CE approved stent is the Viabahn (W. L. Gore and Associates, Flagstaff, AZ) which is a heparinbonded stent known for its ability to inhibit the development of in-stent restenosis [21-24]. The aim of our study is to investigate whether a Viabahn stent sandwiched between drug eluted stents will help to minimise edge neointimal hyperplasia.

\section{Materials and Methods}

2.1. Patients. Between late September 2010 and July 2012, a total of 46 PTA procedures were performed with an intention to employ the sandwich stents technique (described later). Firstly, referrals to our vascular interventional unit were on the presentation of a long segment femoropopliteal chronic total occlusive disease. Disease is graded from moderate to severe reflecting patients' life-style altering intermittent claudication and ischaemic rest pain according to Rutherford and Trans-Atlantic Inter-Society Consensus-II (TASC-II) $[1,3]$. Data collection included clinical presentation, demographics, and radiological and associated comorbidities; see Table 1. In all cases, concurrent morbidities at the time of the procedure decision have precluded their surgical suitability rendering them a high risk for any surgical intervention by the consensus of surgical and anaesthetic consultants.

2.2. Sandwich Technique. Our new technique consists of an interventional procedure performed in the majority of cases through an ipsilateral vascular access by puncturing the common femoral artery, followed by the introduction of a 7F introducer sheath commonly used in all cases (BRITE TIP, Cordis, FL, USA). Angiographic assessment by the interventional radiologist is based on antegrade lower extremity runs with various angles/projections. This is followed by an angioplasty attempt to break through the occluded lesion to achieve an adequate inflow of blood supply through either an intraluminal or a subintimal route. By large, intraluminal recanalization could be challenging to achieve and occasionally followed by successful subintimal new recanalization. If the latter route is not stented immediately, it is likely to be threatened with reocclusion. Thus, a decision to deploy the stents through the new subintimal channel using the sandwich technique is justified. After establishing recanalization through intraluminal or subintimal route, the new track is predilated with balloon to permit deployment of the heparinbonded Viabahn (W. L. Fore Associates, Flagstaff, AZ) stent(s) to cover the entire recanalized occlusion distance if possible, followed by two drug-eluting Zilver PTX stents
TABLE 1: Patients' demographics, associated comorbidities, aspirin, and subgrouping according to Rutherford and TASC-II classification.

\begin{tabular}{lcc}
\hline Parameter & $N=46$ patients & Percentage (\%) \\
\hline Age & Ave. age $=75.3$ & \\
$>65$ & 37 & $80.5 \%$ \\
Gender & & \\
Male & 33 & $71.7 \%$ \\
Female & 13 & $28.3 \%$ \\
Comorbidities & & \\
Smoking & 22 & $47.8 \%$ \\
IHD & 25 & $54.3 \%$ \\
Stroke & 9 & $19.6 \%$ \\
DM & 14 & $30 \%$ \\
HTN & 33 & $71.7 \%$ \\
High cholesterol & 27 & $58.7 \%$ \\
Aspirin & 33 & $71.7 \%$ \\
TASC-II & & \\
C & 21 & $45.7 \%$ \\
D & 25 & $54.3 \%$ \\
Rutherford & & \\
3 & 13 & $28.3 \%$ \\
4 & 11 & $23.9 \%$ \\
5 & 18 & $39.1 \%$ \\
6 & 4 & $8.7 \%$ \\
\hline
\end{tabular}

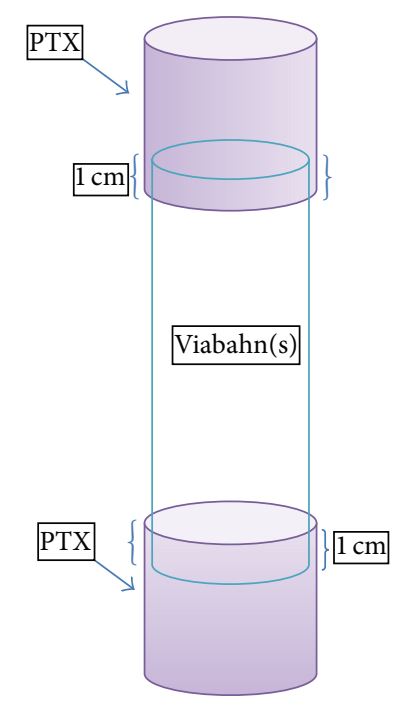

FIGURE 1: Diagrammatic illustration of the procedure technique, highlighting a distance of $1 \mathrm{~cm}$ overlap between two types of deployed stents at the proximal and distal ends.

(Cook Medical, Bloomington, IN), one on either end (proximal and distal ends) of the Viabahn stent(s) with an overlap of $1 \mathrm{~cm}$ (Figure 1).

Heparin was administered perioperatively in all cases using the intra-arterial route with the appropriate doses according to the national guidelines and the institution protocol. Haemostasis is achieved by using either an Angioseal 
system (St. Jude Medical, St. Paul, MN) or manual compression. All patients were started if not already on antiplatelet therapy (e.g., aspirin and/or clopidogrel).

2.3. Followup, Data Analysis, and Ethics. A retrospective cohort review was conducted of all available radiological reports/images, outpatient clinic letters, postprocedure duplex scans, subsequent records of multidisciplinary team (MDT) discussions, and/or reintervention. Follow-up points were set at $0,3,12,18$, and 36 months. However, followup in few cases has evidently exceeded that by further 6 months after their annual review (i.e., 18 or 40 months of followup). Mid-term patency is defined by confirmed continuous patency between end-points at zero to 18 months after intervention (which includes short- until mid-term). Long-term patency is then defined by being more than 18 and up to 36 months (long-term) provided that a continuous clinical improvement requiring no further interventions is evident from the health records. A full database containing demographics, presenting manifestations, TASC classification, procedure details, and outcome as well as comorbidities, was analysed to calculate log-rank tests and Kaplan-Meier curves using Statistical Package for Social Sciences (SPSS, Chicago, IL) version 21.0. Both stent brands are already licensed at our institution and in the UK for SFA occlusion angioplasty.

Ethically, the proposed technique and cohort analysis were approved and categorised by "Clinical Audit Governance" terms and conditions as a clinical evaluation of service improvement since edge neointimal hyperplasia after PTAs has been previously noted with covered stents. Procedure steps, potential outcomes, and complications were always explained and discussed with the patients to enable the obtainment of an informed consent prior to the procedure. The completed written consents (type 1) and WHO Checklists were also cosigned by both the clinical team and the performing interventional radiologist and then scanned and digitally uploaded to our reporting radiology systems. In emergency cases with irreversible incapacity or documented mental incompetence, the performing interventionalist and referring vascular surgeon cosigned the appropriate consent form (type 4) in patients' best interest preprocedure date and following witnessed and documented discussion with their relatives and/or carers.

\section{Results}

A total of 46 PTAs were performed, and the majority were male patients representing $72 \%$ of cases (33/46). Average age was 75 years with standard deviation of 10.97 (range 53-94 years). Found comorbidities and Rutherford score results are summarised in Table 1. Only two cases required bilateral access in which only one side had the sandwich technique (severely occluded side). Two main classes of TASC-II classifications were identified in the whole study, TASC-C $(n=21)$ and TASC-D $(n=25)$.

Intraluminal recanalization of the occluded segment was successfully achieved in 20 cases. The subintimal route with the occasional use of the outback device (Outback LTD
Re-Entry Catheter, Cordis, FL, USA) was successful in 26 patients by breaking into the extra-anatomical layer to rejoin the true lumen distally; one subintimal entry sandwich technique case did not require the reentry device. A total of 44 Viabahn and 74 PTX stents were used to treat 46 lower limb angioplasties (Table 2).

The mean length of arterial peripheral disease was $28.35 \mathrm{~cm}$ (standard deviation $=13.15 \mathrm{~cm}$ ). The Viabahn mean length was $16.96 \mathrm{~cm}$ using three available length options (10, 15 , and $25 \mathrm{~cm}$ ) in the diameters of 5 or $6 \mathrm{~mm}$. The mean PTX length was $8.64 \mathrm{~cm}$ with a range of lengths varying from 4 to $24 \mathrm{~cm}$ and used diameters ranging from 5 to $7 \mathrm{~mm}$. In several occasions, using more than one Viabahn stent was necessary to cover the whole occluded distance. Standard balloon dilation prior to stent deployment was undertaken in most cases, especially with the subintimal routes using a range of 4-6 $\mathrm{mm}$ balloon diameters.

Only $n=27$ patients received a typical "Sandwich" technique to their occluded SFA with confirmed proximal and distal PTX stents sandwiching Viabahn stent(s) in between (Figures 2(a)-2(c)). It is this subset of patients, cohort (A), that authors have decided to compare with the atypical subset, cohort (B), from the point of view of their mid- and long-term patency in Tables 3(a) and 3(b). In cohort (B), atypical procedure to the sandwich technique but consisting of conventional PTA steps was noted in the remaining 19 cases. In those cases, a (documented) decision was taken to perform the conventionally accepted angioplasty using various types and sizes of deployed stents, such as by deploying a single/two PTX stent(s) alone or Viabahn stent(s) alone or one of each but not in any combination that resembles the sandwiching configuration as described in the methodology above (Figure 1). Therefore, this particular cohort (B) has been excluded from the final statistical analysis for being a conventional angioplasty with various steps and intravascular components causing heterogeneity in their methodology.

The mid-term patency of cohort (A) shows that $41 \%$ (11/27) of the typically sandwiched stents have occluded within the first month after procedure, five of which had occluded just in the first week. Out of these occluded cases, ten cases were classed as TASC-D disease (39\%). During longterm followup, one occluded case (TASC-D) underwent successful redo-PTAs and remains patent so far within twelve $(45 \%, 12 / 27)$ patent sandwich technique cases in cohort (A). Following the original intervention, two TASC-C and three TASC-D patients died in the first month; hence, their postprocedure patency was not formally assessed. This remained the total mortality up till the end of followup.

In cohort (B), TASC-C is noted to be the predominant grade of disease severity in this atypical group (90\%, 17/19). Four deaths were the only recorded mortality in the first six months. Occlusion rate is $26-31 \%$ during the whole study (5 cases during mid-term updated to 6 in long-term followup out of 19), and all were TASC-C cases. The remaining 52\% $(10 / 19)$ patent cases have lost one case during the longterm followup due to occlusion leading to a subsequent limb amputation. Among the occluded cases in cohort (B), three had no further reinterventions, while another two occluded with subsequent femoropopliteal bypass surgery. In general, 


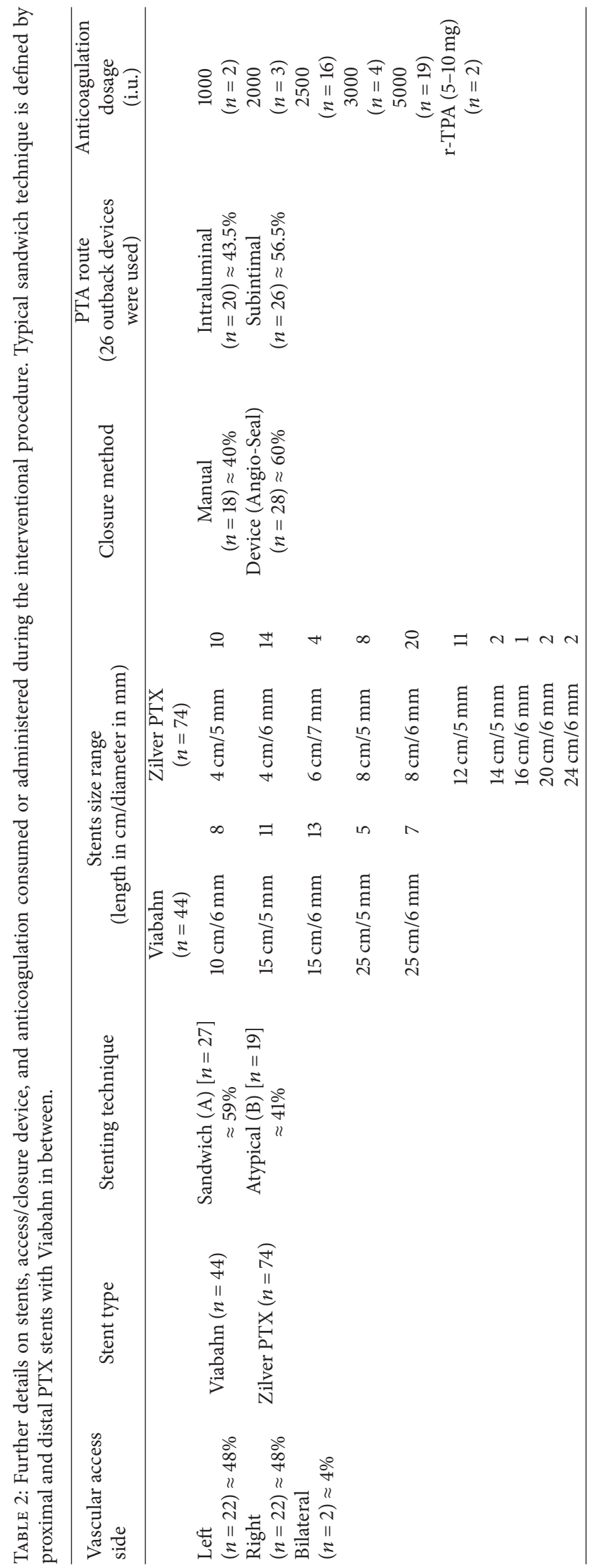




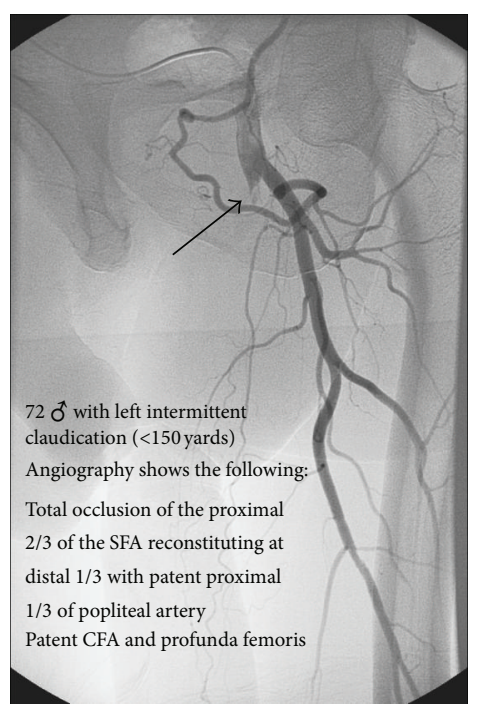

(a)

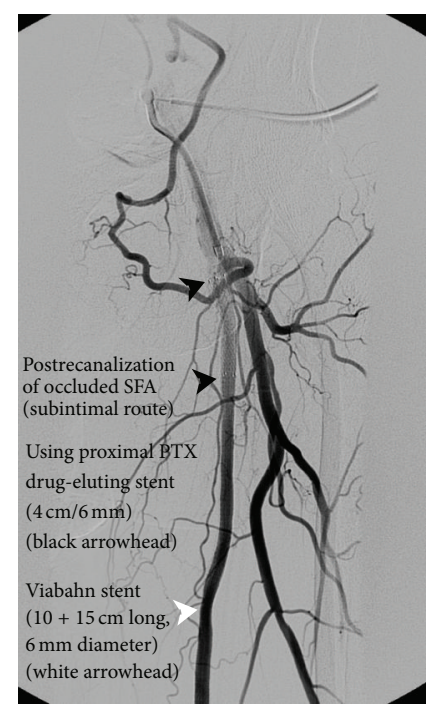

(b)

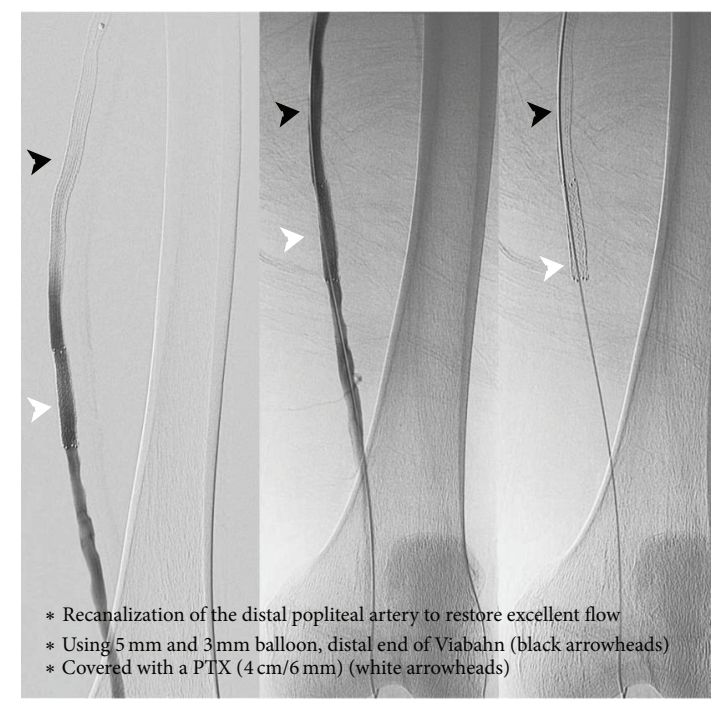

(c)

FIGURE 2: Selected images from the angiographic runs during the sandwich technique procedure for a 72-year-old male patient with left-sided flush SFA chronic total occlusion (proximal two-thirds).

two cases occluded (one of each cohort) at the end of midterm followup and each had a redo-PTA which remains patent in just one case (sandwich technique case).

All deaths observed during the whole period of followup were due to unrelated causes to the interventional procedure. No postprocedural complications were observed in either cohort of patients. Average in-hospital admission for all patients was 24 hours.

Further statistical analysis of cohort (A) was performed by comparing several known variables within the cohort (Tables 1 and 2). The most significant factors were plotted on 3 different Kaplan-Meier curves by adjusting their time of followup to 1200 days after procedure (Figures 3(b)-3(d)). The first curve shows that more than $50 \%$ of total stented limbs using the sandwich technique remained patent during the whole study, with reocclusion preponderance for TASC-D
(Figure 3(a)). Albeit a smaller proportion, TASC-C subset itself has demonstrated a considerably sustained favourable outcome with $50 \%$ stents patency (Figure 3(b)).

Additionally, the site of vascular (entry point) recanalization showed positive effect on overall stent patency. As depicted in Figure 3(c), the subintimal route seems to provide a lower rate of postprocedural occlusion compared to the intraluminal route. From the observed cohorts' demographics, aspirin compliance, as stated in the patients notes and from their electronic drug charts, was the only patient factor demonstrating a relative state of prolonged patency without occlusion (Figure 3(d)). Albeit not statistically significant (log rank test $P=0.25$ ), comorbidities analysis revealed some observed advantage of the sandwich technique in the subset of patients with documented type II diabetes mellitus $(n=14$, Figure 3(e)). 
TABLE 3: (a) and (b) summarise the findings of mid- and long-term followup for the typical sandwich technique, cohort (A), and the atypical cases, cohort (B). During the mid-term followup, only 5 patients died in cohort (A) and 4 patients died in cohort (B). No further mortalities were observed over the long term.

(a)

\begin{tabular}{lcccc}
\hline $\begin{array}{l}\text { Cohort (A) followup } \\
\text { (Typical sandwich, } n=27)\end{array}$ & TASC II & Vessel patency & Occlusion & $n=$ \\
\hline Mid-term & $n=$ & $n=$ & 1 & Deceased \\
{$[0-18$ months $]$} & {$[\mathrm{C}] n=6$} & 3 & 10 & 3 \\
\hline Long-term & {$[\mathrm{D}] n=21$} & 8 & 1 & None \\
{$[18-36$ months $]$} & {$[\mathrm{C}] n=4$} & 3 & 9 & None \\
\hline
\end{tabular}

(b)

\begin{tabular}{lcccc}
\hline Cohort (B) followup & TASC II & Vessel patency & Occlusion & $n=$ \\
(Atypical technique, $n=19)$ & $n=$ & $n=$ & 5 & Deceased \\
\hline Mid-term & {$[\mathrm{C}] n=17$} & 8 & 4 \\
{$[0-18$ months $]$} & {$[\mathrm{D}] n=2$} & 2 & None & None \\
\hline Long-term & {$[\mathrm{C}] n=13$} & 7 & 6 & None \\
{$[18-36$ months $]$} & {$[\mathrm{D}] n=2$} & 2 & None \\
\hline
\end{tabular}

\section{Discussion}

In this retrospective study, a total of 46 patients underwent lower limb angioplasty; 27 of them received a new proposed technique (sandwich technique) of stenting without any immediate or late complications. The intention of this technique as a final resort in salvaging chronically diseased limbs is to alleviate symptoms, improve circulation, and heal ulcerations. During such time, patients' nonsurgical status due to reversible comorbidities might alter favourably.

Early results of our new interventional technique were initially encouraging in early management of CTO of the superficial femoral artery, especially in TASC-C subset. While the majority of patients had consistently received the new technique by sandwiching the Viabahn stent(s) between proximal and distal PTX stent(s), another group of diseased limbs was just suitable for a conventional angioplasty (atypical technique) using one stent type or the other. This was partly because of distal extension of disease behind the knee joint and/or proximal extension into the common or external iliac arteries. In those particular cases, a different individualised approach (i.e., stenting and/or ballooning) was applied as indicated and decided based on a discussion with the patient(s), involved clinical team, and the operating interventionalist (corresponding author), wherever possible. A main limitation noted in this study is the irregularities in follow-up time intervals from procedure date until seen and reviewed by the clinical team. However, towards the end of the long-term followup, the outcome of all case was completed and analysed.

Our mortality analysis of the causes of death indicates that the new procedure had not immediately or indirectly contributed to any of these causes. Admittedly, there is an extra cost (approximately $20 \%$ added to total cost of conventional PTA with one stent type) in short term which is offset by reducing the risk of secondary intervention should neointimal hyperplasia develop causing reocclusion and recurring/worsening symptomatology.

As neointimal hyperplasia is the main culprit in restenosis after angioplasty, histological specimen before or after adopting the new technique did not seem feasible to assess for recurrent occlusive disease. This remains another limitation of this observational study, which focuses on assessing patency first while anticipating that the stents combination would synergically provide a practical preventive measure of any further reocclusion due to neointimal hyperplasia.

It is possible that the results as presented in our followup data are not too promising on the long run. However, as a limb salvage procedure in TASC-C, it is worthwhile reporting the observed potential benefits, especially with early to mid-term results. While statistical significance was not observed in the Kaplan-Meier curves and log-rank tests, those observations do rely on an important message. This new technique is relatively pointing to an apparent improved patency in patients compliant with aspirin and/or triple therapy, predominantly type II diabetics with TASC-C CTO. Equally, it is quite essential to state that, due to the small statistical sampling, those very results should be interpreted with caution.

The sandwich technique has been shown to effectively offer symptoms relief and reduce hospital stay in the first instance. Currently, postprocedural heparin infusions are not a usual practice at our institution because heparin is only given intra-arterially during the procedure. Thus, it is not feasible to demonstrate and compare the real added or adversarial effects of postoperative heparin infusions in bare metal stenting versus the sandwich technique. Nevertheless, perhaps a larger randomised study with a prospective systematic planning may yield the definite evidence on the efficacy of this different technique.

This evidence is envisaged to be similarly important, not only to the patients but also to the medical device industry, 


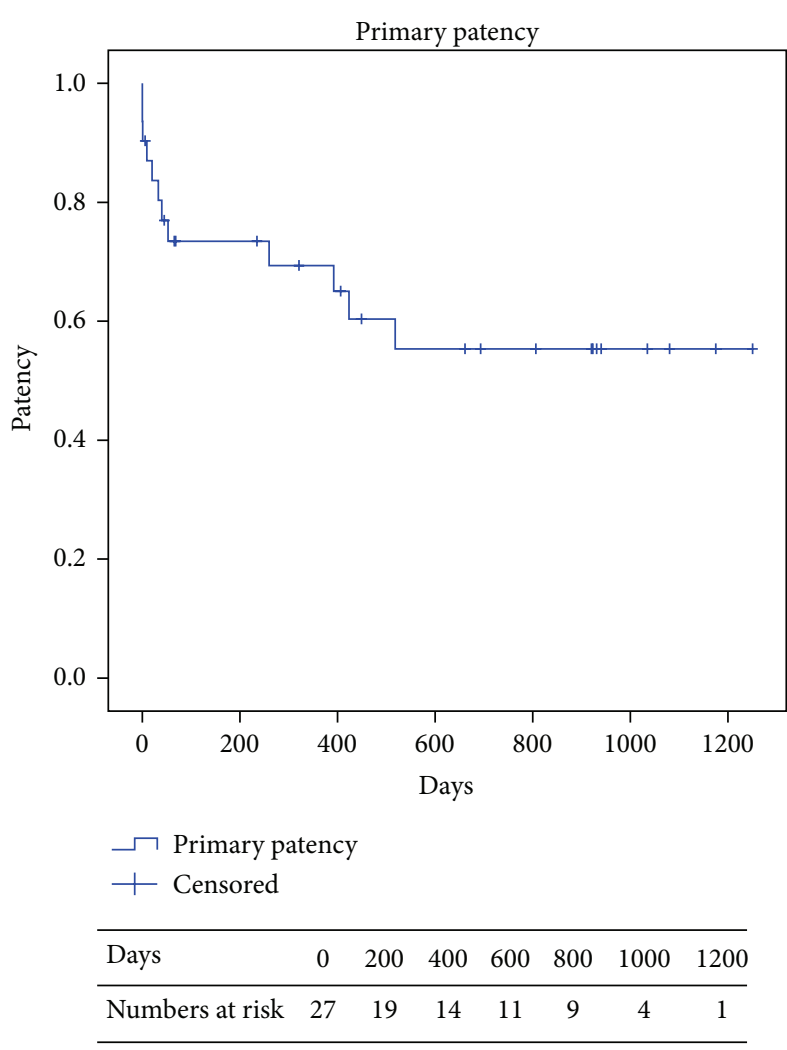

(a)

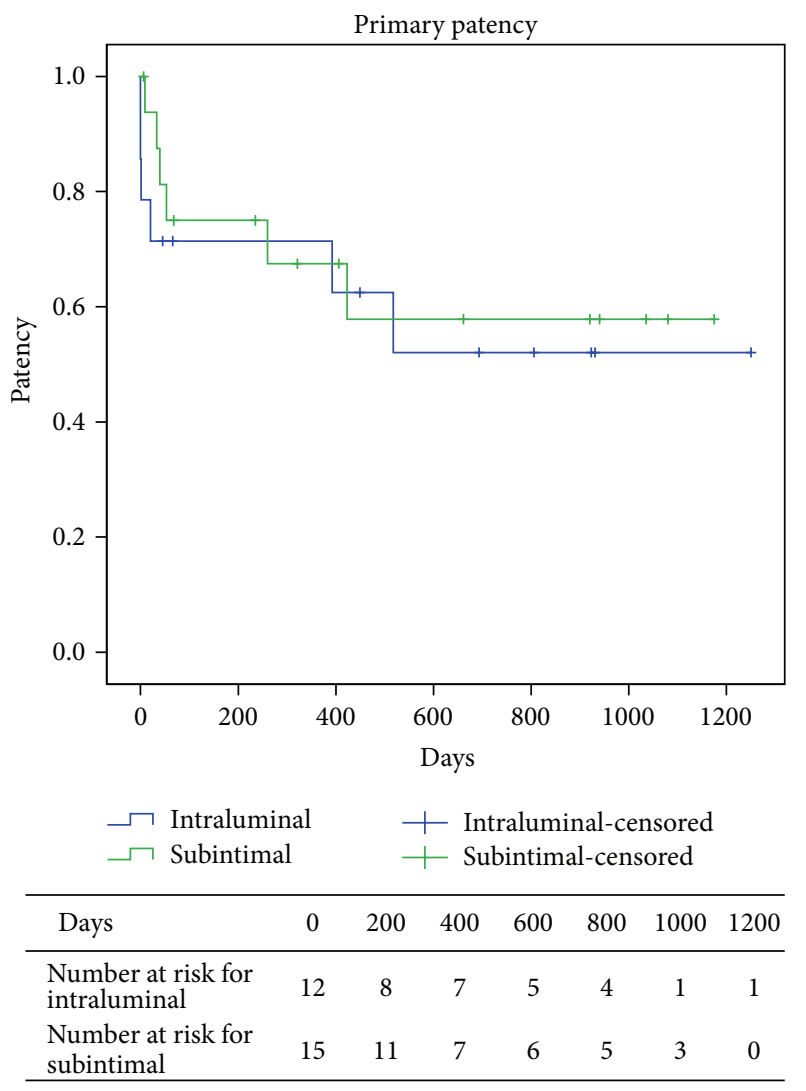

(c)

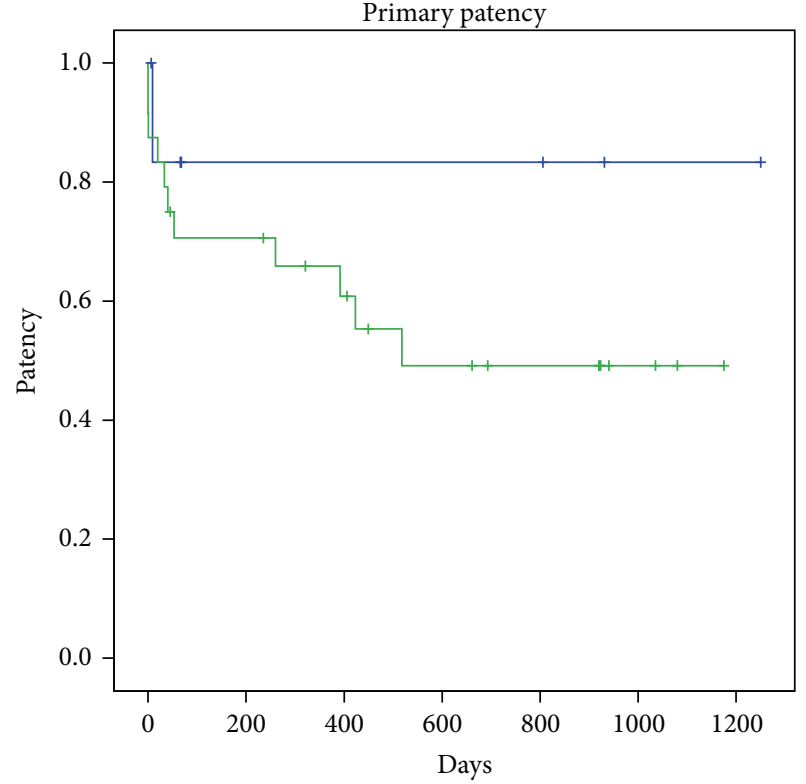

TASC

\begin{tabular}{|c|c|c|c|c|c|c|c|}
\hline $\begin{array}{l}\neg \mathrm{C} \\
\neg \mathrm{D}\end{array}$ & & & $\begin{array}{l}\text { ensor } \\
\text { ensor }\end{array}$ & & & & \\
\hline Days & 0 & 200 & 400 & 600 & 800 & 1000 & 1200 \\
\hline Number at risk for TASC-C & 6 & 3 & 3 & 3 & 3 & 0 & 0 \\
\hline Number at risk for TASC-D & 21 & 16 & 11 & 8 & 6 & 3 & 1 \\
\hline
\end{tabular}

(b)

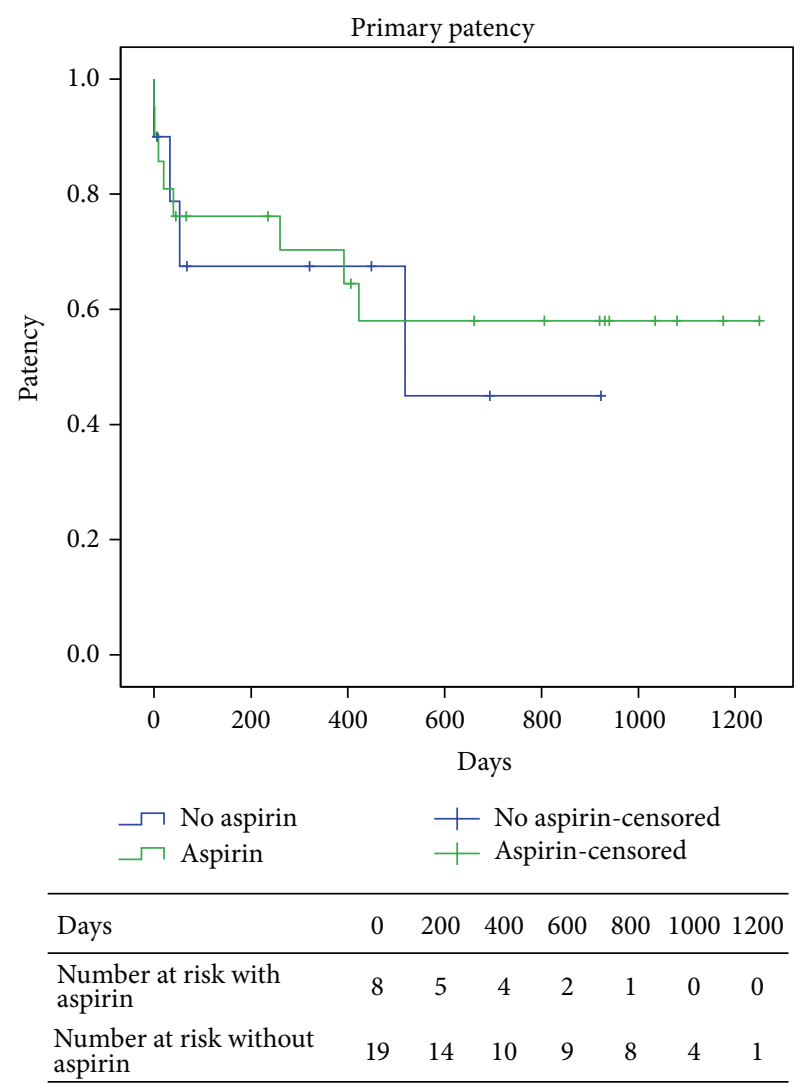

(d)

Figure 3: Continued. 


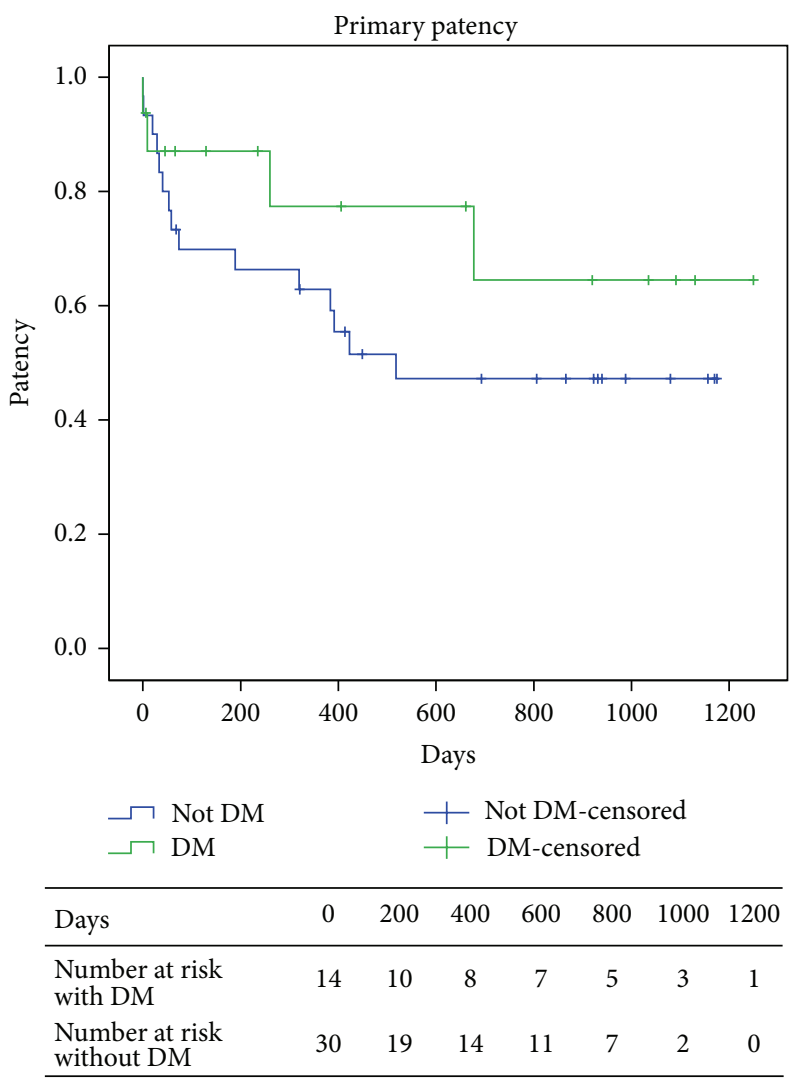

(e)

Figure 3: (a) Kaplan-Meier curve for overall mid- and long-term ( $y$-axis) patency for sandwich technique cases, cohort (A), plotted against days after procedure ( $x$-axis); + indicates censored data. Censored data indicate that observational period was cut off before the occurrence of a significant event (in this case stent occlusion). (b) Kaplan-Meier curve for mid- and long-term patency according to TASC-II classification of femoropopliteal lesions treated by sandwich technique. Log rank test $(P=0.3)$. + indicates censored data. (c) Kaplan-Meier curve for midand long-term patency according to route of recanalization in cohort $(\mathrm{A})$. Log rank test $(P=0.7)$. indicates censored data. (d) Kaplan-Meier curve for mid- and long-term patency with or without regular aspirin in cohort $(\mathrm{A})$. Log rank test $(P=0.8)$. + indicates censored data. (e) Kaplan-Meier curve for primary patency in patients with and without type II diabetes mellitus (DM). Log rank test, $P=0.25 .+$ indicates censored data.

who may consider joining the clinical applications of these two stents into one product and ultimately one function. The problem then may rise from a potential mixture of utilised biomaterials, drug pharmacology, and/or device-device interactions due to unpredicted biochemical interaction between heparin and paclitaxel in one single deployment. Hopefully this concept could be the seed to promising experimentations in current trials at the manufacturers' R\&D laboratories, leading the way to $\mathrm{CE}$ and/or FDA regulated approval before it can be tested on human populations. In addition, joining these stents in a single design and deployment may cut down procedure time to conserve costs and national health resources, while the synergetic benefit of the combination is under continuous system and safety checks.

\section{Conclusions}

To accomplish the hoped results of this new technique in the future, the authors believe that prospective randomization of a larger cohort requires a well-structured methodology of careful patient selection and robust follow-up protocols which entails regular checks by duplex ultrasound and clinical assessments.

\section{Disclosure}

A brief oral presentation presenting the study outcome for this technique has won the first prize at Controversies \& Updates in Vascular Surgery Congress (CACVS) 2013 in Paris, France. An abstract was also presented to CIRSE 2013 in Barcelona, Spain.

\section{Conflict of Interests}

All authors confirm that there are no financial interests that might benefit from the publication of the submitted paper. There are no other competing interests relevant to publication. 


\section{References}

[1] L. Norgren, W. R. Hiatt, J. A. Dormandy, M. R. Nehler, K. A. Harris, and F. G. R. Fowkes, "Inter-society consensus for the management of peripheral arterial disease (TASC II)," Journal of Vascular Surgery, vol. 45, no. 1, supplement, pp. S5-S67, 2007.

[2] G. Kuhan, S. Abisi, B. D. Braithwaite, S. T. R. MacSweeney, S. C. Whitaker, and S. B. Habib, "Early results with the use of heparin-bonded stent graft to rescue failed angioplasty of chronic femoropopliteal occlusive lesions: TASC D lesions have a poor outcome," CardioVascular and Interventional Radiology, vol. 35, no. 5, pp. 1023-1028, 2012.

[3] J. A. Dormandy and R. B. Rutherford, "Management of peripheral arterial disease (PAD). TASC Working Group. TransAtlantic Inter-Society Consensus (TASC)," Journal of Vascular Surgery, vol. 31, no. 1, part 2, pp. S1-S296, 2000.

[4] S. H. Duda, B. Pusich, G. Richter et al., "Sirolimus-eluting stents for the treatment of obstructive superficial femoral artery disease: six-month results," Circulation, vol. 106, no. 12, pp. 15051509, 2002.

[5] J. R. Laird, B. T. Katzen, D. Scheinert et al., "Nitinol stent implantation versus balloon angioplasty for lesions in the superficial femoral artery and proximal popliteal artery: twelvemonth results from the RESILIENT randomized trial," Circulation: Cardiovascular Interventions, vol. 3, no. 3, pp. 267-276, 2010.

[6] M. Schillinger, S. Sabeti, C. Loewe et al., "Balloon angioplasty versus implantation of nitinol stents in the superficial femoral artery," The New England Journal of Medicine, vol. 354, no. 18, pp. 1879-1888, 2006.

[7] M. Schillinger, S. Sabeti, P. Dick et al., "Sustained benefit at 2 years of primary femoropopliteal stenting compared with balloon angioplasty with optional stenting," Circulation, vol. 115, no. 21, pp. 2745-2749, 2007.

[8] M. D. Dake, G. M. Ansel, M. R. Jaff et al., "Paclitaxel-eluting stents show superiority to balloon angioplasty and bare metal stents in femoropopliteal disease: twelve-month zilver PTX randomized study results," Circulation: Cardiovascular Interventions, vol. 4, no. 5, pp. 495-504, 2011.

[9] B.-N. Nguyen, M. F. Conrad, J. M. Guest et al., "Late outcomes of balloon angioplasty and angioplasty with selective stenting for superficial femoral-popliteal disease are equivalent," Journal of Vascular Surgery, vol. 54, no. 4, pp. 1051.el-1057.e1, 2011.

[10] H. Krankenberg, M. Schlüter, H. J. Steinkamp et al., "Nitinol stent implantation versus percutaneous transluminal angioplasty in superficial femoral artery lesions up to $10 \mathrm{~cm}$ in length: the Femoral Artery Stenting Trial (FAST)," Circulation, vol. 116, no. 3, pp. 285-292, 2007.

[11] P. Dick, H. Wallner, S. Sabeti et al., "Balloon angioplasty versus stenting with nitinol stents in intermediate length superficial femoral artery lesions," Catheterization and Cardiovascular Interventions, vol. 74, no. 7, pp. 1090-1095, 2009.

[12] O. Schlager, P. Dick, S. Sabeti et al., "Long-segment SFA stenting-the dark sides: in-stent restenosis, clinical deterioration, and stent fractures," Journal of Endovascular Therapy, vol. 12, no. 6, pp. 676-684, 2005.

[13] B. Scheller, U. Speck, C. Abramjuk, U. Bernhardt, M. Böhm, and G. Nickenig, "Paclitaxel balloon coating, a novel method for prevention and therapy of restenosis," Circulation, vol. 110, no. 7, pp. 810-814, 2004.
[14] S. Cassese, R. A. Byrne, I. Ott et al., "Paclitaxel-coated versus uncoated balloon angioplasty reduces target lesion revascularization in patients with femoropopliteal arterial disease: a meta-analysis of randomized trials," Circulation: Cardiovascular Interventions, vol. 5, no. 4, pp. 582-589, 2012.

[15] G. Tepe, S. Schmitmeier, and T. Zeller, "Drug-coated balloons in peripheral arterial disease," EuroIntervention, vol. 7, supplement K, pp. K70-K76, 2011.

[16] S. H. Duda, M. Bosiers, J. Lammer et al., "Drug-eluting and bare nitinol stents for the treatment of atherosclerotic lesions in the superficial femoral artery: long-term results from the SIROCCO trial," Journal of Endovascular Therapy, vol. 13, no. 6, pp. 701-710, 2006.

[17] J. Lammer, M. Bosiers, T. Zeller et al., "First clinical trial of nitinol self-expanding everolimus-eluting stent implantation for peripheral arterial occlusive disease," Journal of Vascular Surgery, vol. 54, no. 2, pp. 394-401, 2011.

[18] M. Bosiers, P. Peeters, J. Tessarek, K. Deloose, and S. Strickler, "The Zilver PTX single arm study: 12-month results from the TASC C/D lesion subgroup," Journal of Cardiovascular Surgery, vol. 54, no. 1, pp. 115-122, 2013.

[19] M. D. Dake, G. M. Ansel, M. R. Jaff et al., "Sustained safety and effectiveness of paclitaxel-eluting stents for femoropopliteal lesions: 2-year follow-up from the zilver PTX randomized and single-arm clinical studies," Journal of the American College of Cardiology, vol. 61, no. 24, pp. 2417-2427, 2013.

[20] T. Zeller, M. D. Dake, G. Tepe et al., "Treatment of femoropopliteal in-stent restenosis with paclitaxel-eluting stents," JACC: Cardiovascular Interventions, vol. 6, no. 3, pp. 274-281, 2013.

[21] T. Jahnke, R. Andresen, S. Müller-Hülsbeck et al., "Hemobahn stent-grafts for treatment of femoropopliteal arterial obstructions: midterm results of a prospective trial," Journal of Vascular and Interventional Radiology, vol. 14, no. 1, pp. 41-51, 2003.

[22] J. Lammer, T. Zeller, K. A. Hausegger et al., "Heparin-bonded covered stents versus bare-metal stents for complex femoropopliteal artery lesions: the randomized VIASTAR trial (viabahn endoprosthesis with propaten bioactive surface [VIA] versus bare nitinol stent in the treatment of long lesions in superficial femoral artery occlusive disease)," Journal of the American College of Cardiology, vol. 62, no. 15, pp. 1320-1327, 2013.

[23] R. R. Saxon, A. Chervu, P. A. Jones et al., "Heparin-bonded, expanded polytetrafluoroethylene-lined stent graft in the treatment of femoropopliteal artery disease: 1-year results of the VIPER (viabahn endoprosthesis with heparin bioactive surface in the treatment of superficial femoral artery obstructive disease) trial," Journal of Vascular and Interventional Radiology, vol. 24, no. 2, pp. 165-173, 2013.

[24] P. J. Geraghty, M. W. Mewissen, M. R. Jaff, and G. M. Ansel, "Three-year results of the VIBRANT trial of VIABAHN endoprosthesis versus bare nitinol stent implantation for complex superficial femoral artery occlusive disease," Journal of Vascular Surgery, vol. 58, no. 2, pp. 386.e4-395.e4, 2013. 


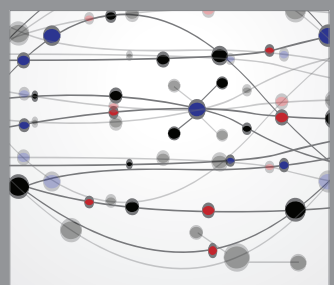

The Scientific World Journal
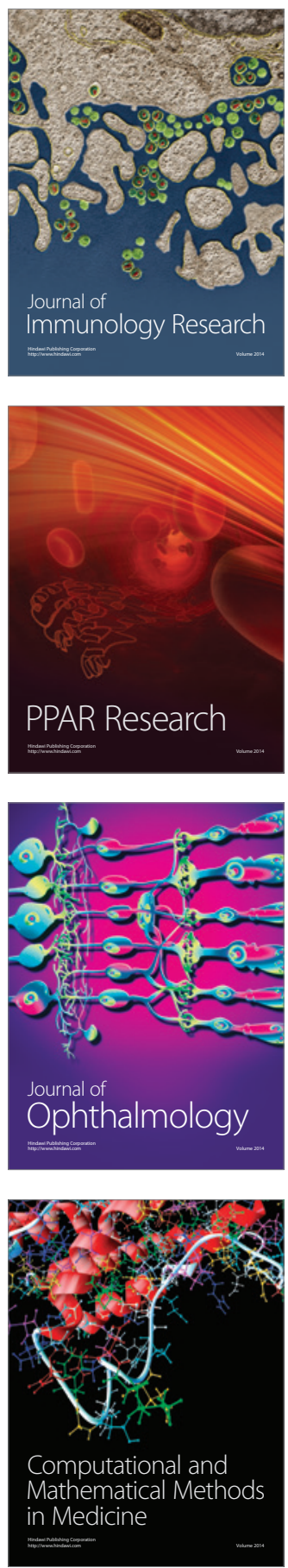

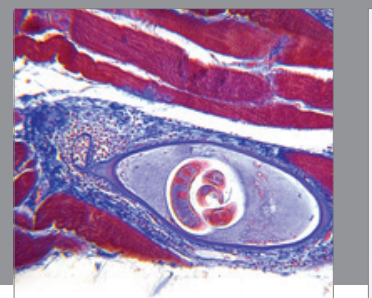

Gastroenterology

Research and Practice
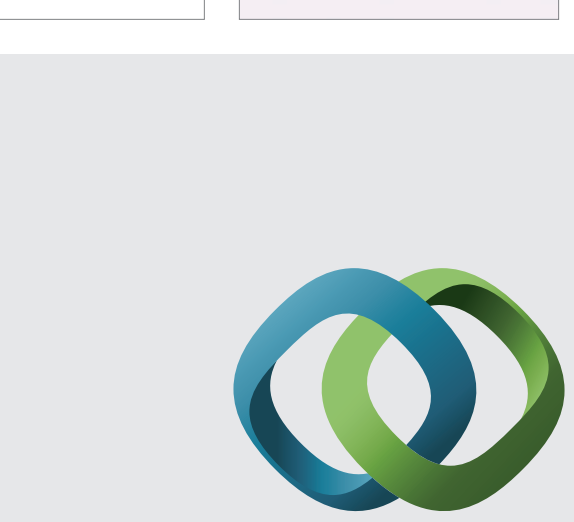

\section{Hindawi}

Submit your manuscripts at

http://www.hindawi.com
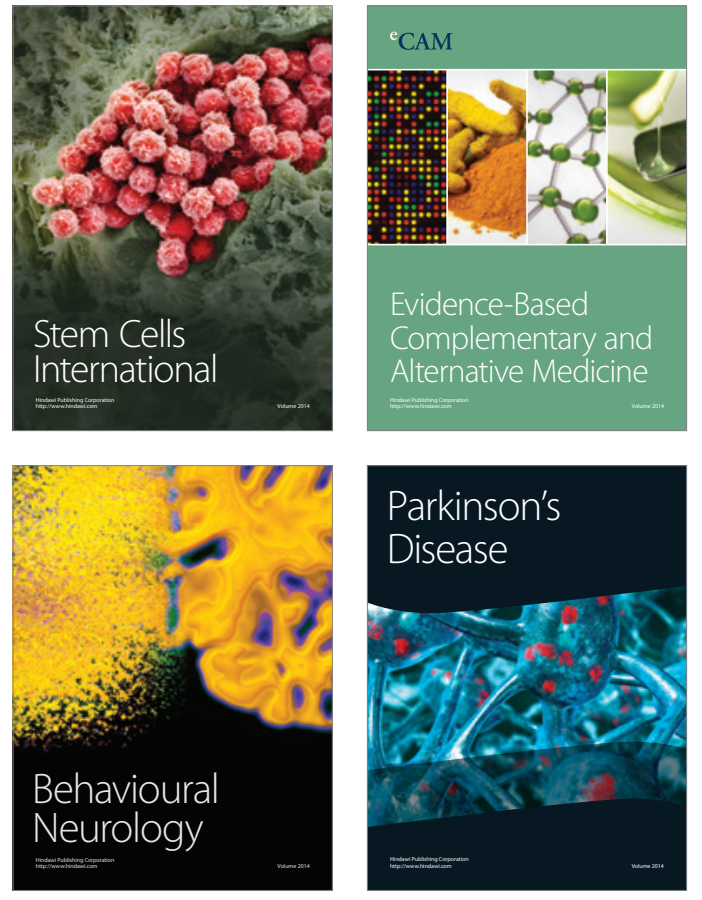
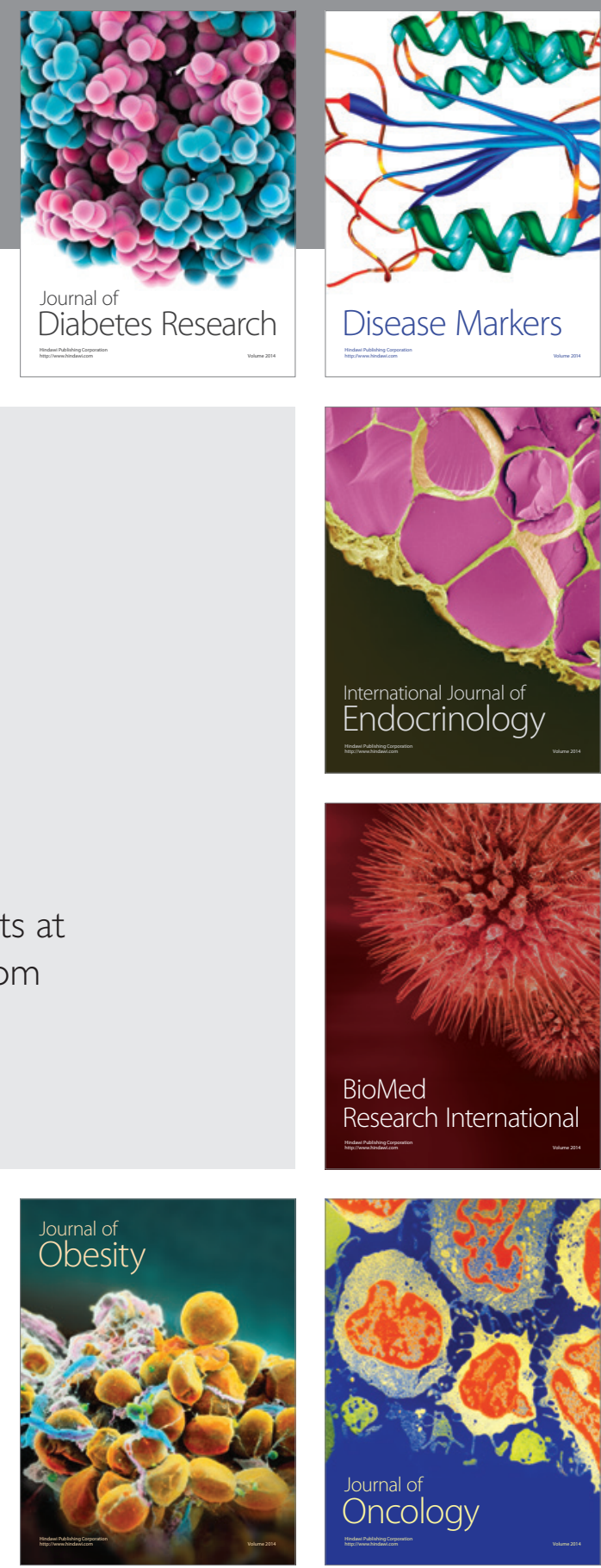

Disease Markers
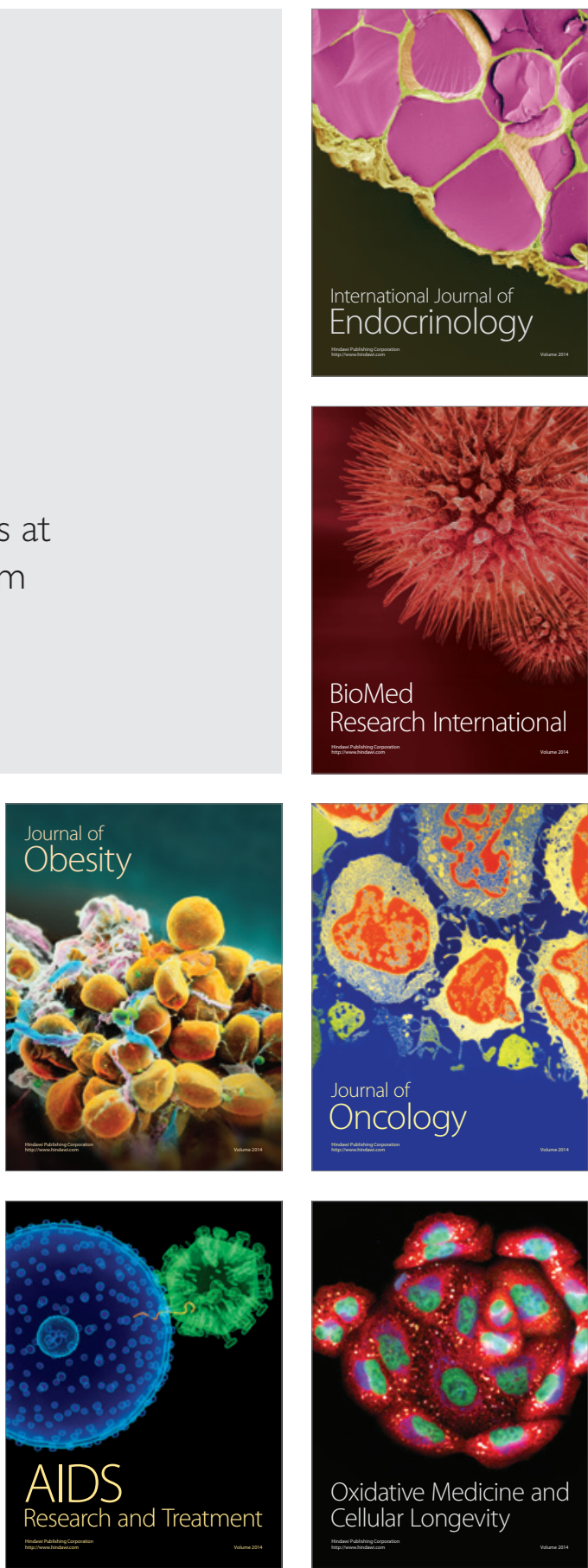